\title{
Influence of Defects on the Electronic Structures of Bilayer Graphene
}

\author{
Ken Kishimoto*, Susumu Okada \\ Graduate School of Pure and Applied Sciences, University of Tsukuba, 1-1-1 Tennodai, \\ Tsukuba, Ibaraki 305-8571, Japan
}

\begin{abstract}
Based on first-principles total-energy calculation, we investigate the electronic structures of bilayer graphene, one of which layers possesses atomic or topological defects, to explore the possibility of band gap engineering of graphene by means of physisorption of defective graphene. Our calculations show that the pristine graphene layer possesses a finite energy gap between bonding and antibonding $\pi$ states because of the potential undulation caused by the other graphene layer with defects. We also found that the gap values strongly depend on the defect species and their mutual arrangement with respect to the pristine layer.
\end{abstract}

Keywords: Bilayer graphene, Band gap, Electronic structure, Interlayer hybridization

\section{Introduction}

Following the synthesis of graphene from bulk graphite [1, 2, 3, 4], graphene is attracting much attention in the field of low-dimensional science and nanotechnology $[5,6]$. Graphene is the ultimate version of atomic layered materials, consisting of hexagonally bonded $\mathrm{sp}^{2} \mathrm{C}$ atoms. This geometric structure endows graphene with unique physical and chemical properties, which allow us to observe the unusual quantum Hall effect and remarkable carrier mobility $[7,8]$. From a technological view, because of their sheet structure with

*TEL/FAX: +81-298535600 (ext. 8233)/+81-298535924

Email addresses: kkishimoto@comas.frsc.tsukuba.ac.jp (Ken Kishimoto), sokada@comas.frsc.tsukuba.ac.jp (Susumu Okada) 
atom thickness and the peculiar electronic structure, graphene is regarded as an emerging material for various devices in the near future, such as sensing devices, semiconducting devices, and transparent electrodes, despite the difficulties of device fabrication and fragility of their electronic properties in hybrid structures in devices. For instance, graphene adsorbed onto $\mathrm{SiO}_{2}$ and $\mathrm{HfO}_{2}$ is not a metal but a semiconductor with an energy gap of approximately a few tens of meV because of the electrostatic potential modulation arising from the electrons and nuclei of substrates $[9,10]$. Metal surfaces also substantially modulate the electronic structure of graphene because of the orbital hybridization [11].

The electronic structures of graphene are sensitively dependent on imperfections such as atomic defects, topological defects, and edges. It has been demonstrated that graphene with defects is either a metal or a semiconductor, depending on the defect structure and arrangement [12, 13]. Edges with zigzag shapes lead to spin polarization at edge atomic sites because of the flat dispersion bands arising from the topologically induced edge localized states $[14,15,16,17]$. Furthermore, topological defects also induce peculiar electronic states at/near the Fermi level because of the symmetry breaking of the $\mathrm{AB}$ bipartite network of graphene $[18,19,20]$. In addition to the imperfections introduced into the atomic covalent network, interlayer stacking arrangements of graphene thin films also lead to interesting variations in their electronic structures because of the sizable overlap between $\pi$ states of adjacent layers. For instance, bilayer graphene is either a metal or a semiconductor, depending on the interlayer stacking arrangement [21]. Rhombohedral graphene thin films possess flat dispersion bands around the $\mathrm{K}$ point, leading to ferrimagnetic spin ordering on their outermost atomic layers $[22,23,24]$. These facts imply that the electronic structure of graphene is tunable by controlling the intralayer imperfections and the interlayer stacking arrangements.

In the present work, we aim to investigate the electronic structure of bilayer graphene, one of which layers possesses atomic or topological defects, and to explore the possibility of the electronic structure tuning of graphene by forming such bilayer structures, based on first principles total energy calculations in the framework of density functional theory. Our calculations show that all bilayer structures do not possess Dirac cones at or near the Fermi level, but finite energy gap in their $\pi$ electronic states even though one of two layers retains a hexagonal covalent network. The energy gap between $\pi$ and $\pi^{*}$ states belonging to the pristine layer depends on the defect species 
and arrangements. We find that the spatial modulation of the electrostatic potential on a pristine layer as a result of the other layer with defects leads to electronic structure modulation. Further analysis of the wave functions distribution shows substantial interlayer hybridization leading to modulation of band dispersions from that obtained from the simple sum of those of each constituent.

\section{Calculation methods and structural models}

(a)

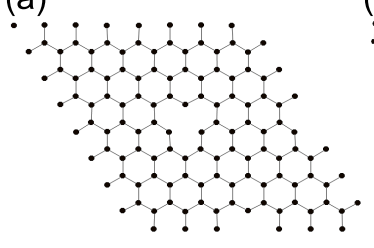

(b)

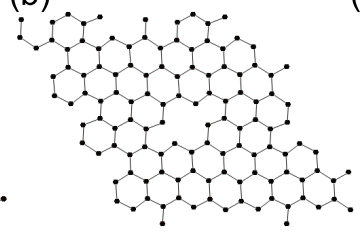

(c)

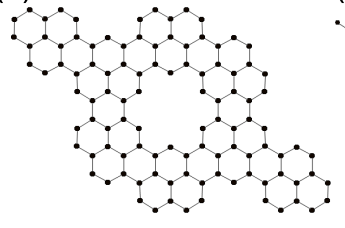

(d)

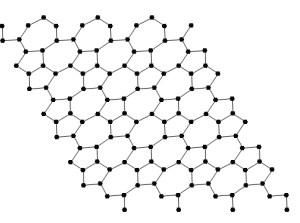

Figure 1: Optimized atomic structures of defective graphene with (a) monovacancies, (b) divacancies, (c) hexagonal vacancies (V6), and (d) topological defects.

All calculations were performed based on the framework of density functional theory $[25,26]$ using the Simulation Tool for Atom Technology (STATE) package [27]. We used local density approximation [28, 29] to describe the exchange-correlation potential among the interacting electrons. An ultrasoft pseudopotential generated by the Vanderbilt scheme was used to describe the interaction between electrons and ions [30]. The valence wave functions and charge density were expanded in terms of the plane-wave basis set with cutoff energies of 25 and $255 \mathrm{Ry}$, respectively. To exclude unphysical dipole interaction with periodic images normal to the graphene layers, we imposed an open boundary condition normal to the layers using the effective screening medium (ESM) method [31]. In the present work, we consider bilayer graphene, one of which layers contains atomic or topological defects. As for the defects, we consider monovacancy, divacancy, hexagonal vacancy, and Stone-Wales topological defects per $4 \times 4$ lateral unit cell of pristine graphene (Fig. 1). Although the lateral lattice parameter strongly depends on the defect species and densities, we fixed the lateral parameter as $9.84 \AA$ corresponding to that of $4 \times 4$ lateral cell of pristine graphene to satisfy the commensurability condition between pristine and defective layers. Atomic 
structures of the bilayer graphene were optimized under an interlayer spacing of $3.4 \AA$ with an $\mathrm{AB}$ stacking arrangement until the force acting on each atom is less than $1.33 \times 10^{-3} \mathrm{HR} / \mathrm{au}$. Integration over the Brillouin zone was carried out using an equidistance mesh of $4 \times 4 \times 1 \boldsymbol{k}$ points for bilayer graphene with $4 \times 4$ lateral periodicity, which corresponds to the $16 \times 16 \times 1$ $\boldsymbol{k}$-point grid for conventional cell of graphene leading to the sufficient convergence in the electronic structures. It should be noted that the inter-layer spacing of graphene depend on the defect species and densities. Indeed, our LDA calculations on the bilayer graphene with $4 \times 4$ lateral cell show that the optimum spacings between pristine and defective layers are 3.33, 3.33, 3.29, and $3.32 \AA$ for the monovacancy, divacancy, V6 vacancy, and topological defects, respectively.

\section{Results and Discussion}

(a)

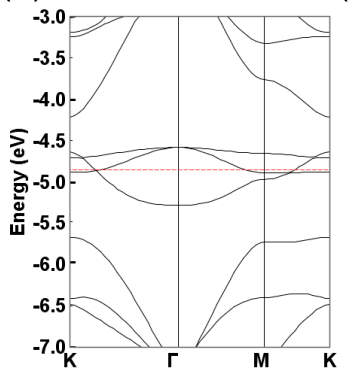

(b)

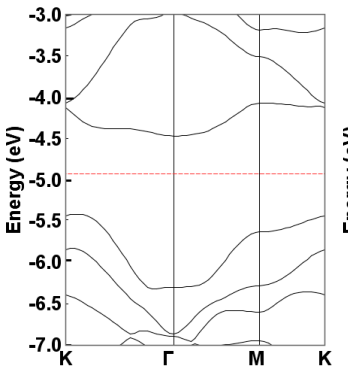

(c)

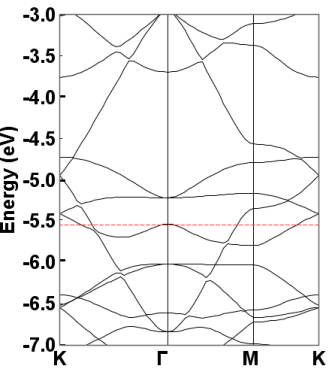

(d)

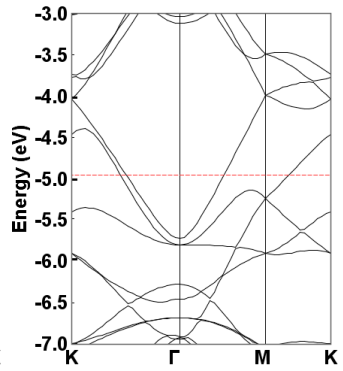

Figure 2: Electronic structures of graphene with (a) monovacancies, (b) divacancies, (c) hexagonal vacancies (V6), and (d) topological defects. Energies are measured from that of the vacuum level. Dotted horizontal lines denote the Fermi level.

Figure 2 shows the electronic structures of monolayer graphene with atomic and topological defects. In all cases, the electronic energy bands of graphene with defects are significantly modulated from those of pristine graphene by introducing the defects. In some cases, the linear dispersion bands are absent, while relatively small dispersion bands emerge near the Fermi level. For graphene with monovacancies [Fig. 2(a)], three states cross the Fermi level in the energy gap between two dispersive bands. These small dispersion bands are associated with the $\sigma$ state of dangling bonds at the vacancy, while the bands with large dispersion correspond to the bonding and antibonding $\pi$ states. In this case, because the $\mathrm{C}-\mathrm{C}$ network does not satisfy 
the Kekule pattern, the $\pi$ states have finite energy gaps at the K point. For graphene with divacancies [Fig. 2(b)], the sheet is a semiconductor with an indirect energy gap of $1 \mathrm{eV}$ in which dispersive and flat bands emerge around the gap associated with $\pi$ and defect-induced states, respectively [Fig. 2(b)]. In this case, because of the formation of pentagonal rings around the defects, dangling bond states with $\sigma$ nature are absent at the Fermi level. In the case of graphene with hexagonal vacancies (V6) [Fig. 2(c)], the $\pi$ network topologies satisfy the perfect Kekule pattern, so that they possess a pair of dispersive bands with nearly linear dispersion above the Fermi level. In addition to the nearly linear dispersion bands, several relatively flat dispersion bands emerge around the Fermi level because of the dangling bond states around the atomic defects. Two-dimensional $\mathrm{C}$ sheets consisting of fused pentagons and heptagons also have linear dispersion bands above the Fermi level [Fig. 2(d)]. In this case, because the network consists only of $\mathrm{C}$ atoms with three-fold coordination, the dangling bond states are absent.

Figure 3(a) shows the electronic structure of bilayer graphene, one of which layers contains monovacancies per $4 \times 4$ lateral periodicity. In this case, the atomic defect is located above the hollow sites of the pristine graphene. As shown in Fig. 3(a), the bilayer graphene does not possess linear dispersion bands associated with $\pi$ and $\pi^{*}$ states, although the system contains pristine graphene. In contrast, five states emerge near the Fermi level: three of the five states have small dispersions exhibiting the same characteristics as the defect-induced states in the monolayer graphene with monovacancies shown in Fig. 2(a). Indeed, these three states are distributed in the inplane direction, exhibiting the dangling bond nature of $\sigma$ states at the defect [Fig. 4(a)]. The other two states, which are absent in the monolayer graphene with monovacancies, have substantial dispersion and exhibit extended $\pi$ nature. These two states have quadric dispersion around the $\mathrm{K}$ point with the energy gap of $0.64 \mathrm{eV}$, in contrast to the linear dispersion of $\pi$ states in pristine graphene. Although the states are associated with the pristine layer, the states on sheets exhibit hybridized $\pi$ nature between layers.

It is necessary to investigate the influence of the relative position of the defects to the pristine layer on the electronic structure of bilayer graphene. Figure 3(b) shows the electronic structure of graphene adsorbed on the graphene with monovacancies located above the atomic site of the pristine layer, as mentioned above. In this case, we also find two dispersive bands above and below the Fermi level that are absent in the monolayer graphene with monovacancies, in addition to the three flat dispersion bands associated with the 
defects. These dispersive bands have a finite gap of $0.07 \mathrm{eV}$ at the $\mathrm{K}$ point, which is considerably narrower than that found in the case of the other relative position of monovacancies to the pristine layer. In this case, the $\pi$ states associated with the pristine layer slightly hybridize with the $\pi$ states of the defective layer compared with the other stacking pattern [Fig. 4(b)]. Therefore, the amount of hybridization between the layers affects the gap of the $\pi$ states of pristine graphene by the physisorption of the graphene layer with defects. Furthermore, the relative position of the defect to the atomic arrangement of the pristine layer decisively affects the electronic structure of the bilayer system. In addition to the relative position, the defect density also affects the electronic structure of pristine layer. With increasing the defect density by four times (monovacancy per $2 \times 2$ lateral cell), the gap decrease by $0.4 \mathrm{eV}$ than the gap for the case of $4 \times 4$ cell. Thus, the defect density also affects the electronic structure of the pristine layer of bilayer graphene.

Figure 3(c) shows the electronic structure of bilayer graphene consisting of pristine and defective layers containing divacancies. By comparing the band structure of the bilayer graphene consisting of pristine and defective layers to that of the isolated graphene with divacancies, we find two dispersive bands near the Fermi level. These two dispersive bands still have quadric dispersion with a finite gap of $0.07 \mathrm{eV}$ at the $\mathrm{K}$ point, although the states are expected to possess $\pi$ and $\pi^{*}$ nature belonging to the pristine layer. In this case, the $\pi$ states with the quadric dispersion relation above and below the Fermi level slightly hybridize with the $\pi$ states belonging to the defective graphene, as shown in Fig. 4(c). This interlayer hybridization leads to the small energy gap between the $\pi$ states of the pristine layer.

By adsorbing the graphene with V6 vacancies on pristine graphene with $0.34 \mathrm{~nm}$ interlayer spacing, we find that the interlayer interaction modulates the electronic structures of both pristine graphene and graphene with V6 defects. As shown in Fig. 3(d), the electronic structure of the bilayer graphene, one of which layers contains V6 vacancies, does not possess linear dispersion bands around the Fermi level, although both layers are expected to possess a pair of linear dispersion bands near the Fermi level: four dispersive bands with quadric dispersion relations emerge near the Fermi level. These states cross the flat dispersion bands associated with $\sigma$ states at the defect. Two of the four states, labeled $\pi$ and $\pi^{*}$, are distributed on the pristine layer with $\pi$ electron nature [Fig. 4(d)]. These states also have a finite energy gap of $0.18 \mathrm{eV}$ at the $\mathrm{K}$ point. In contrast, the remaining two states are distributed 
on the defective layer with $\pi$ nature and have a gap of $0.22 \mathrm{eV}$. In this case, interlayer hybridization is moderate, so that the gap is also moderate.

Our calculations showed that graphene has a finite energy gap between $\pi$ and $\pi^{*}$ states by adsorbing on defective graphene, irrespective of the vacancy species. Thus, finally, we investigate the possibility of the similar electronic structure modulation of graphene adsorbed on an $\mathrm{sp}^{2} \mathrm{C}$ network of fused pentagons and heptagons as a structural model of graphene with topological defects. Figure 3(e) shows the electronic structure of bilayer $\mathrm{C}$ sheets consisting of pristine graphene and a $\mathrm{C}$ sheet comprising fused pentagons and heptagons. We found two additional dispersive bands near the Fermi level that correspond to the $\pi$ and $\pi^{*}$ states of the pristine graphene layer. Although there is no atomic defect in both layers, the dispersive band corresponding to the bonding and antibonding $\pi$ states has a finite energy gap of $0.15 \mathrm{eV}$. In this case, hybridization of the wave function between layers is marginal [Fig. 4(e)].

Table 1: Energy gap between $\pi$ and $\pi^{*}$ states and the electrostatic potential variation of pristine graphene layer adsorbed on the defect layer.

\begin{tabular}{ccc}
\hline & $\pi-\pi^{*}$ gap $[\mathrm{eV}]$ & $\Delta \mathrm{V}[\mathrm{eV}]$ \\
\hline \hline Monovacancy (on hollow site) & 0.64 & 0.11 \\
Monovacancy (on atomic site) & 0.07 & 0.14 \\
Divacancy & 0.07 & 0.12 \\
Hexagonal vacancy & 0.18 & 0.43 \\
Topological defect & 0.15 & 0.04 \\
\hline
\end{tabular}

Table I summarizes the energy gap between $\pi$ and $\pi^{*}$ states of pristine graphene adsorbed on the defective graphene. In all cases, the pristine layer possesses a finite energy gap between $\pi$ and $\pi^{*}$ states, although the layer retains its honeycomb network. The calculated energy gaps range from 0.07 to $0.64 \mathrm{eV}$, depending on the mutual stacking arrangement and the defect species. Thus, the defective graphene can affect the electronic structures of pristine graphene by adsorbing onto them with appropriate interlayer spacing.

To elucidate the physical origin of the gap opening on graphene influenced by the graphene with defects, we investigate the modulation of the electrostatic potential on the pristine graphene by adsorbing on the graphene with defects from the isolated monolayer graphene. Figure 5 shows contour plots of the modulation of the electrostatic potential just above the pristine 
graphene layer under the bilayer structure with the defective layer. The potential modulation is calculated by taking the difference between the electrostatic potential of bilayer graphene and the sum of the electrostatic potentials of each constituent layer. As shown in Fig. 5, the electrostatic potential is spatially modulated along the lateral direction at the nanometer scale for all cases. This fact indicates that the electrons in the pristine layer feel this potential undulation, causing the finite energy gap for the $\pi$ electrons in pristine graphene adsorbed on graphene with defects. As shown in Table I, the energy gap does not correlate with the electrostatic potential modulation on the pristine layer. We found that the potential modulation depends on the size of the atomic vacancies: The modulation in V6 is the largest among the five, while that in topological defects is the smallest. Inhomogeneous charge density around the large atomic vacancies may cause large modulation of the electrostatic potential on the pristine layer. The fact indicates that the gap value mainly depends on the amount of the orbital hybridization between defective and pristine layers.

\section{Conclusion}

We studied the electronic structure of bilayer graphene consisting of pristine and defective graphene layers using density functional theory with local density approximation. Our calculations showed that all bilayer graphene no longer possesses pairs of linear dispersion bands at or near the Fermi level, but instead has quadric dispersion bands together with the states associated with the defects, even though one of two layers retains their hexagonal atomic network. The energy gap between the top and the bottom of $\pi$ states belonging to the pristine layer strongly depends on the defect species and arrangement. We also find that the $\pi$ states belonging to the pristine layer more or less hybridize with the electron states in the graphene with defects. The amount of hybridization seems to correlate with the energy gap of the $\pi$ states in the pristine layer. Furthermore, analysis of the electrostatic potential of bilayer graphene revealed that the spatial undulation of the electrostatic potential on the pristine layer arising from the defective layer causes the energy gap between bonding and antibonding $\pi$ states in the pristine layer. Our findings indicate a possible procedure for realizing semiconducting graphene by adsorbing on graphene with defects or by introducing defects in one of two layers of bilayer graphene. Furthermore, the gap is tunable by controlling the defect species and their mutual arrangement with respect to the pristine 
graphene.

\section{Acknowledgments}

This work was supported in part by a Grant-in-Aid for Scientific Research from the Ministry of Education, Culture, Sports, Science and Technology of Japan. Computations were performed on a NEC SX-8/4B at the University of Tsukuba, a NEC SX-9 at the Institute for Solid State Physics, The University of Tokyo, and a NEC SX-Ace at the Cybermedia Center, Osaka University.

\section{References}

[1] K. S. Novoselov, A. K. Geim, S. V. Morozov, D. Jiang, Y. Zhang, S. V. Dubonos, I. V. Grigorieva, and A. A. Firsov, Science, 306 (2004) 666.

[2] A. K. Geim and K. S. Novoselov, Nature Mater. 6 (2007) 183.

[3] I. Forbeaux, J.-M. Themlin, and J.-M. Debever, Phys. Rev. B 58 (1998) 16396.

[4] C. Berger, Z. Song, X. Li, X. Wu, N. Brown, C. Naud, D. Mayou, T. Li, J. Hass, A. N. Marchenkov, E. H. Conrad, P. N. First, and W. A. de Heer, Science, 312 (2006) 1191.

[5] M. S. Dresselhaus and G. Dresselhaus, Adv. Phys. 30 (1981) 139.

[6] A. H. Castro Neto, F. Guinea, N. M. R. Peres, K. S. Novoselov, and A. K. Geim, Rev. Mod. Phys. 81 (2009) 109.

[7] K. S. Novoselov, A. K. Geim, S. V. Morozov, D. Jiang, M. I. Katsnelson, I. V. Grigorieva, S. V. Dubonos, and A. A. Firsov, Nature, 438 (2005) 197.

[8] Y. Zhang, Y.-W. Tan, H. L. Stormer, and P. Kim, Nature, 438 (2005) 201.

[9] N.T. Cuong, M. Otani, and S. Okada, Phys. Rev. Lett. 106 (2011) 106801.

[10] K. Kamiya, N. Umezawa, and S. Okada, Phys. Rev. B 83 (2011) 153413. 
[11] Y. Takagi and S. Okada, Jpn. J. Appl. Phys. 51 (2012) 085102.

[12] Y. Ma, P. O. Lehtinen, A. S. Foster, and R. M. Nieminen, New J. Phys. $6(2004) 68$

[13] M. Igami, K. Nakada, and S. Okada, Synth. Metal 121 (2001) 1233.

[14] M. Fujita, L. Wakabayashi, K. Nakada, and K. Kusakabe, J. Phys. Soc. Jpn. 65 (1996) 1920.

[15] K. Nakada, M Fujita, G. Dresselhaus and M. S. Dresselhaus, Phys. Rev. B 54 (1996) 17954.

[16] Y. Miyamoto, K. Nakada, and M. Fujita, Phys. Rev. B 59 (1999) 9858.

[17] P. Koskinen, S. Malola, and H. Häkkinen, Phys. Rev. Lett. 101 (2008) 115502.

[18] S. Okada, T. Kawai, and K. Nakada, J. Phys. Soc. Jpn. 80 (2011) 013709.

[19] M. Maruyama and S. Okada, Appl. Phys. Express 6 (2013) 095101.

[20] M. Maruyama and S. Okada, Jpn. J. Appl. Phys. 53 (2014) 06JD02.

[21] S. Konabe and S. Okada, J. Phys. Soc. Jpn. 81 (2012) 113702.

[22] M. Koshino and T. Ando, Phys. Rev. B 76 (2007) 085425.

[23] M. Koshino and T. Ando, Phys. Rev. B 77 (2008) 115313.

[24] M. Otani, M. Koshino, Y. Takagi, and S. Okada, Phys. Rev. B 81 (2010) $161403(\mathrm{R})$.

[25] P. Hohenberg and W. Kohn, Phys. Rev. 136 (1964) B864.

[26] W. Kohn and L.J. Sham, Phys. Rev. 140 (1965) A1133.

[27] Y. Morikawa, K. Iwata, and K. Terakura, Appl. Surf. Sci. 169-170 (2000) 11.

[28] J.P. Perdew and A. Zunger, Phys. Rev. B 23 (1981) 5048.

[29] D.M. Ceperley and B.J. Alder, Phys. Rev. Lett. 45 (1980) 566. 
[30] D. Vanderbilt, Phys. Rev. B 41 (1990) 7892.

[31] M. Otani and O. Sugino, Phys. Rev. B 73 (2006) 115407. 
(a)

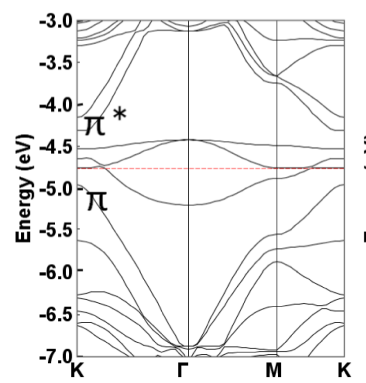

(c)

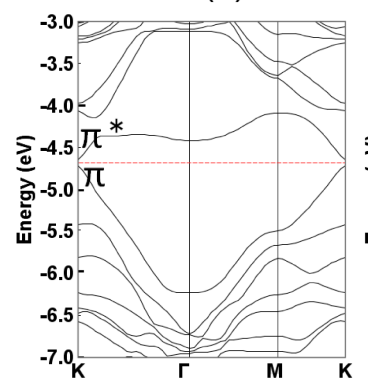

(e)

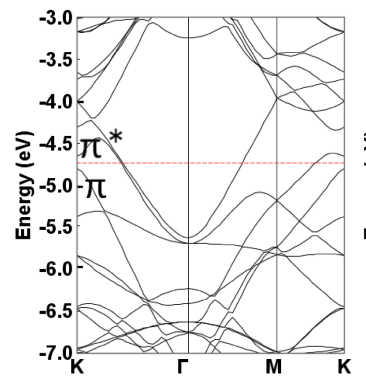

(b)
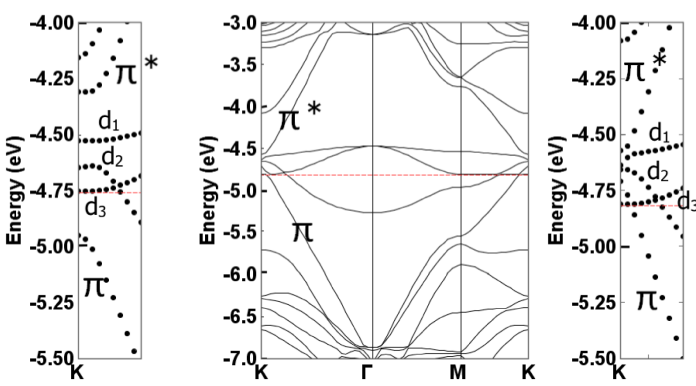

(d)
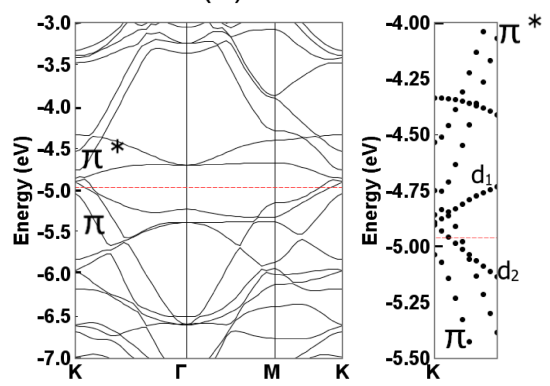

Figure 3: Electronic structures of bilayer graphene, one of which layers contains (a) monovacancies above the hollow sites, (b) monovacancies above the atomic sites, (c) divacancies, (d) hexagonal vacancies, and (e) topological defects. Energies are measured from that of the vacuum level. Red dotted lines denote the Fermi level. In each panel, a right panel is the enlarged view of the electronic structure near the Fermi level. 
(a)

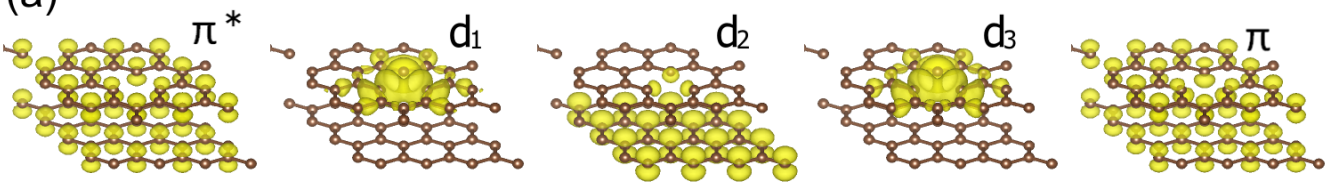

(b)
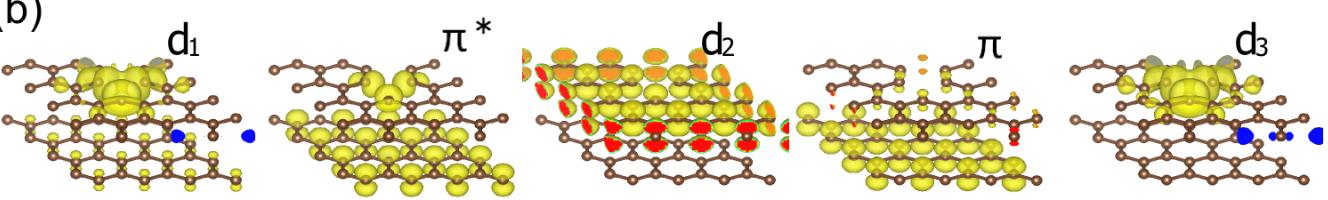

(c)
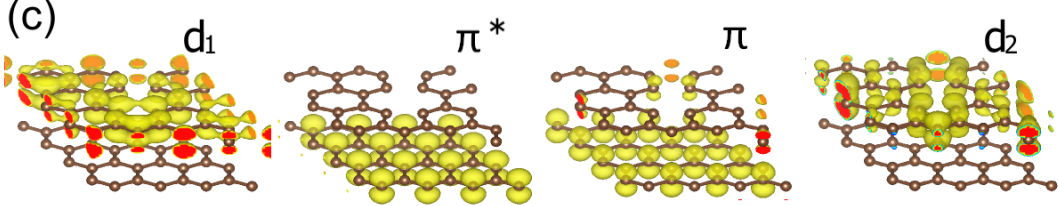

(d)
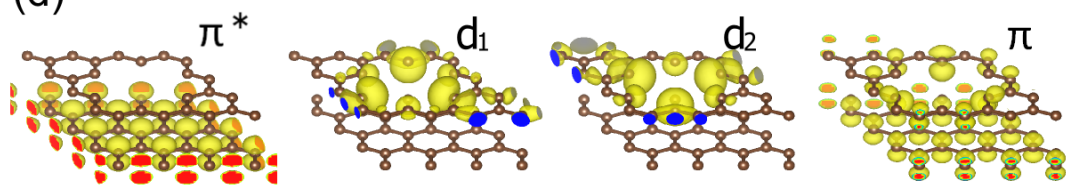

(e)
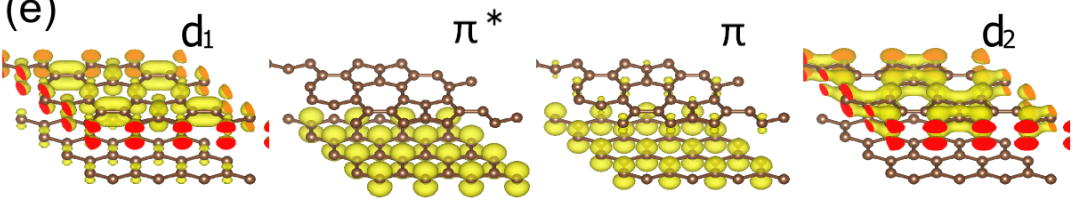

Figure 4: Isosurfaces of squared wave functions at the K point of bilayer graphene, one of which layers contains (a) monovacancies above the hollow sites, (b) monovacancies above the atomic sites, (c) divacancies, (d) hexagonal vacancies, and (e) topological defects. Indexes in each panel correspond to that in the energy band in Fig. 3 
(a)

(b)

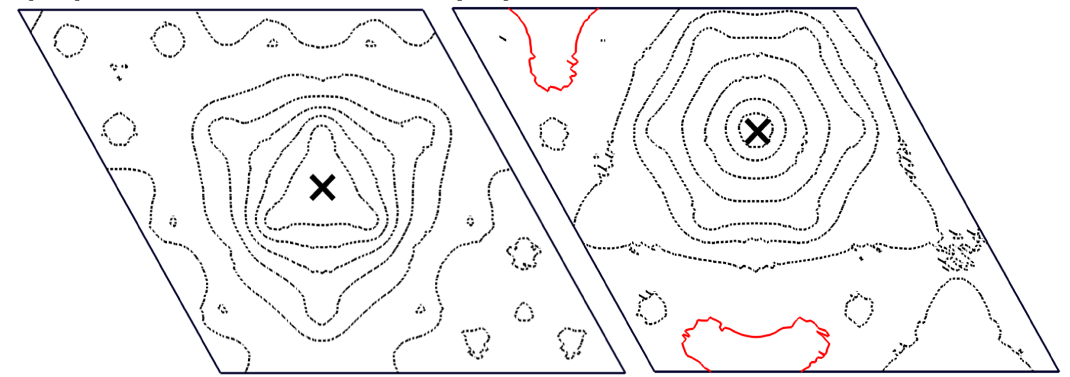

(c)

(d)

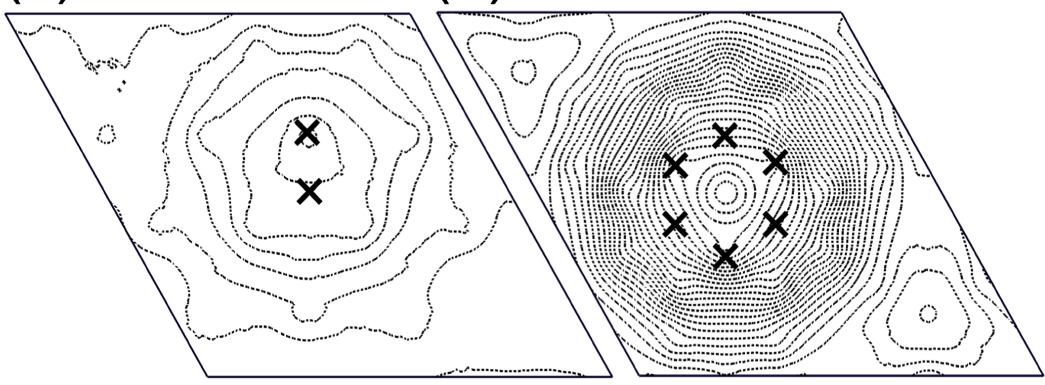

(e)

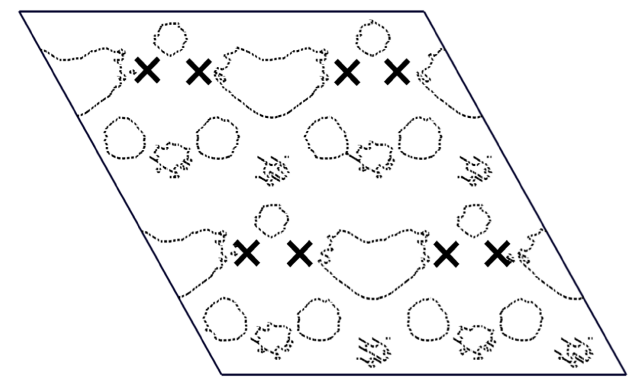

Figure 5: Contour plots of electrostatic potential on the pristine layer of bilayer graphene, one of which layers contains (a) monovacancies above the hollow sites, (b) monovacancies above the atomic sites, (c) divacancies, (d) hexagonal vacancies, and (e) topological defects. Crosses denote the position of vacancies in the defective graphene. Solid and dotted lines denote regions where the potential increases and decreases from that of the isolated graphene without defects, respectively. Each contour corresponds to the potential difference of $0.006 \mathrm{HR}$. 